\title{
Removal of poly-histidine fusion tags from recombinant proteins purified by expanded bed adsorption
}

\begin{abstract}
Enzymatic methods have been used to cleave the $\mathrm{C}$ - or $\mathrm{N}$-terminus polyhistidine tags from histidine tagged proteins following expanded bed purification using immobilized metal affinitychromatography (IMAC). This study assesses the use of Factor Xa and a genetically engineered exopeptidase dipeptidyl aminopeptidase-1 (DAPase-1)for the removal of Cterminusand $\mathrm{N}$-terminus polyhistidine tags, respectively. Model proteins consisting of maltose binding protein (MBP) having a $\mathrm{C}$ - or $\mathrm{N}$-terminal polyhistidine tag were used. Digestion of the hexahistidinetagofMBP-His6 by Factor Xa and HT 15-MBP by DAPase-1 was successful. The time taken to complete the conversion of MBP-HiS6 to MBP was $16 \mathrm{~h}$, as judged by SDS-PAGE and Western blots against anti-His antibody. When the detagged protein was purified using subtractive IMAC, the yield was moderate at $71 \%$ although the overall recovery was high at $95 \%$. Likewise, a yield of $79 \%$ and a recovery of $97 \%$ was obtained when digestion was performed with using "on-column" tag digestion. Oncolumn tag digestion involves cleavage of histidine tag from polyhistidine tagged proteins that are still bound to the IMAC column. Digestion of an N-terminal polyhistidine tag from HT15-MBP $(1 \mathrm{mg} / \mathrm{mL})$ by the DAPase- 1 system was superiorto the results obtained with Factor Xa with a higher yield and recovery of $99 \%$ and $95 \%$, respectively. The digestion by DAPase-1 system was faster and was complete at $5 \mathrm{~h}$ as opposed to $16 \mathrm{~h}$ for Factor Xa. The detagged MBP proteins were isolated from the digestion mixtures using a simple subtractive IMAC column procedure with the detagged protein appearing in the flowthrough and washing fractions while residual dipeptides and DAPase-1 (which was engineered to exhibit a poly-His tail) were adsorbed to the column. FPLC analysis using a MonoS cation exchanger was performed to understand and monitor the progress and time course of DAPase-1 digestion of HT15MBP to MBP. Optimization of process variables such as temperature, protein concentration, and enzyme activity was developed for the DAPase-1 digesting system on HT15-MBP to MBP. In short, this study proved that the use of either Factor Xa or DAPase-1 for the digestion of polyhistidine tags is simple and efficient and can be carried out under mild reaction conditions. (C) 2005 Wiley Periodicals, Inc.
\end{abstract}

Keyword: DAPase; Expanded bed adsorption; Factor Xa; Immobilized metal affinity chromatography; Removal of polyhistidine tails 